\title{
Memory Part 2: The Role of the Medial Temporal Lobe
}

\author{
F.D. Raslau, I.T. Mark, A.P. Klein, J.L. Ulmer, V. Mathews, and (DL.P. Mark
}

$T^{1}$ he medial temporal lobe plays a central role in memory processing and is more than just the hippocampus. ${ }^{1}$ The hippocampal formation, which forms the upper segment of the medial temporal lobe, is a heterogeneous structure consisting of the Ammon horn or Cornus Ammonis (Cornus Ammonis area 1 to Cornus Ammonis area 4) and the dentate gyrus appearing as 2 interlocking $U$ 's on a coronal image. The collapsed portion of the temporal horn of the lateral ventricle forms the superior border of the hippocampal formation. The slightly more dilated lateral aspect of the temporal horn forms the lateral margin, while the ambient cistern is at the medial border. $^{2}$

The parahippocampal gyrus, positioned just inferior to the hippocampus, forms the other major component of the medial temporal lobe (Fig 1). The parahippocampal gyrus is the most medial of the group of 3 gyri that form the inferior surface of the temporal lobe. The collateral sulcus forms the lateral border of the parahippocampal gyrus, while the ambient cistern is at the medial margin. The parahippocampal gyrus can be subdivided into anterior and posterior components. The anterior portion, sometimes referred as the rhinal cortex, ${ }^{3-5}$ consists of medial and lateral parts, the entorhinal (Brodmann area 28) and perirhinal (Brodmann area 35) cortices, respectively. The border between the entorhinal and perirhinal cortices is located at the junction between the inferior surface of the parahippocampal gyrus and the inferior aspect of the collateral sulcus (Fig 2). ${ }^{6,7}$ The superior aspect of the collateral sulcus demarcates the position of the lateral margin of the perirhinal cortex. In other words, the perirhinal cortex forms the medial bank of the collateral sulcus. ${ }^{8}$ The posterior component of the parahippocampal gyrus consists of the parahippocampal cortex, which is not synonymous with the parahippocampal gyrus. The entorhinal, perirhinal, and parahippocampal cortices to-

From the Department of Radiology (F.D.R.), University of Kentucky, Lexington, Kentucky; Morsani College of Medicine (I.T.M.), University of South Florida, Tampa, Florida; and Department of Radiology (A.P.K., J.L.U., V.M., L.P.M.), Medical College of Wisconsin, Milwaukee, Wisconsin.

Please address correspondence to Leighton P. Mark, MD, Froedtert Hospital, Department of Radiology, Neuroradiology Section, 9200 West Wisconsin Ave, Milwaukee, WI 53226; e-mail: Imark@mcw.edu

http://dx.doi.org/10.3174/ajnr.A4169 gether, therefore, make up the parahippocampal gyrus. The subiculum is transitional cortex that bridges the Ammon horn of the hippocampal formation (allocortex) with the parahippocampal gyrus (isocortex). ${ }^{2}$ The subiculum can be further subdivided into the subiculum proper, presubiculum, and parasubiculum.

The organization of the medial temporal lobe suggests a hierarchic format in which information is initially collected through the perirhinal and parahippocampal cortices, passes to the entorhinal cortex, and ultimately reaches the hippocam-

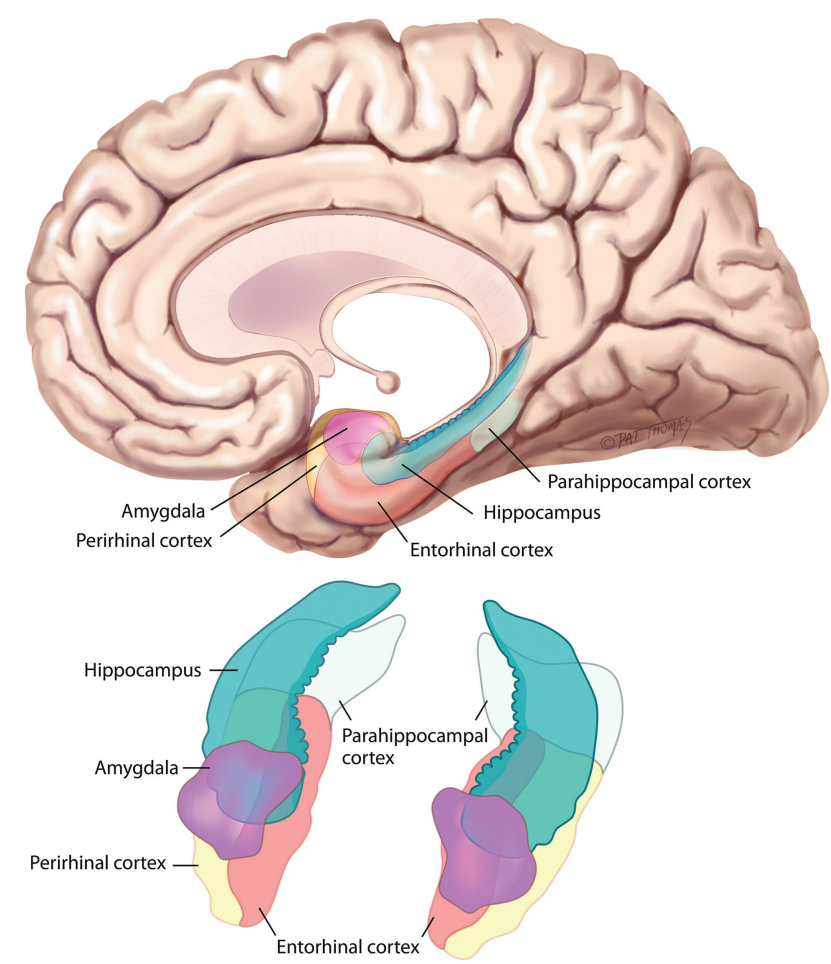

FIG 1. The medial temporal lobe consists of the hippocampal formation (blue-green) superiorly and the parahippocampal gyrus inferiorly. The entorhinal (brown) and perirhinal (yellow) cortices form the medial and lateral components, respectively, of the anterior portion of the parahippocampal gyrus, while the parahippocampal cortex (off-white) forms the posterior portion. Adapted with permission from Purves D, Brannon E, Cabeza R, et al. Principles of Cognitive Neuroscience. Sunderland, MA: Sinauer Associates; 2008. 


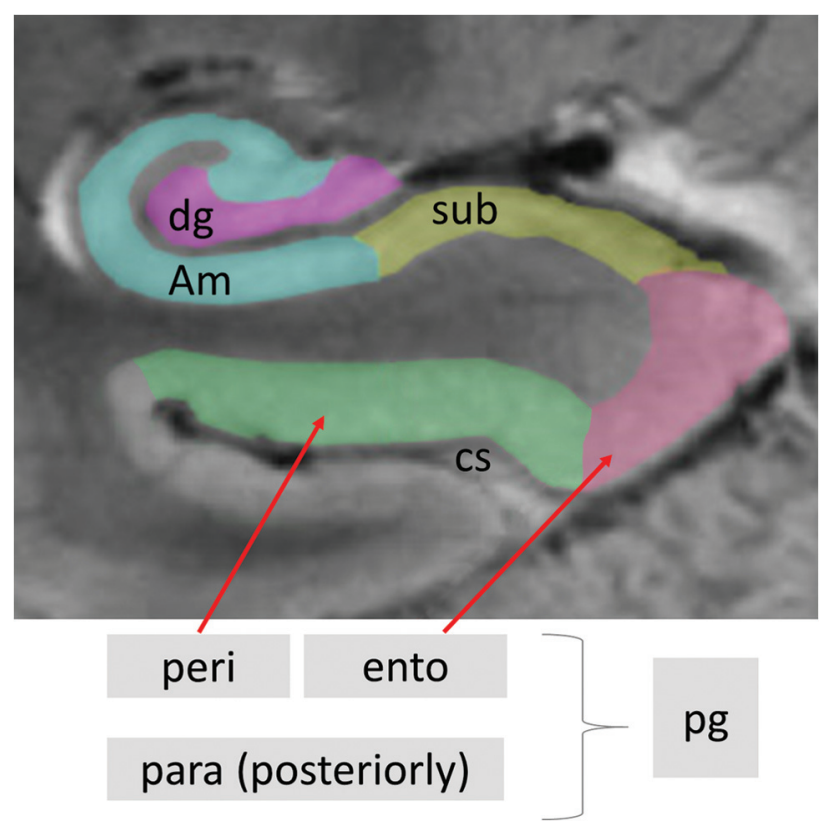

FIG 2. Coronal T2-weighted MR image of the right medial temporal lobe. The Ammon horn (Am) is blue, interlocking with the dentate gyrus (dg) in lavender. The perirhinal cortex (peri) in green is lateral to the entorhinal cortex (ento) in pink. The subiculum (sub) in yellow links the Ammon horn with the entorhinal cortex. Note that the sulcus just lateral to the perirhinal cortex is the collateral sulcus (cs) and the apex or superior most portion of the collateral sulcus marks the position of the lateral margin of the perirhinal cortex, while the inferior aspect of the sulcus marks the border between the perirhinal and entorhinal cortices. The parahippocampal gyus (pg) consists of the perirhinal, entorhinal, and parahippocampal (para) cortices.

pal formation, which forms major output projections via the fornix (Fig 3). ${ }^{9}$ The parahippocampal gyrus, however, does not merely funnel information to the hippocampus. ${ }^{10}$ A large network of connections both within and among the subregions of the parahippocampal gyrus performs extensive information processing on its own and among subregions before any of the information reaches the hippocampus. Exteroceptive (external to the organism) information is processed by the parahippocampal gyrus via the ventral and dorsal streams (Fig 4). The ventral stream from the occipital lobe consists of visual information in terms of object recognition, while the dorsal stream from the parietal lobe carries spatial context information to the parahippocampal gyrus. Interoceptive (internal to the organism) signals carrying information such as emotions and motivation from the medial prefrontal cortex, nucleus accumbens, and amygdala project to the rostral hippocampal formation and rhinal cortex regions. This information from multiple sources is combined and ultimately output via the fornix from the hippocampal formation.

Closer inspection of the medial temporal subregions has revealed additional layers of complexity and organization. The entorhinal cortex, for example, can be seen to have distinctive medial and lateral regions that differ histologically and physiologically. ${ }^{11}$ The medial entorhinal cortex (Brodmann area $28 \mathrm{~b}$ ) is actively involved in the processing of spatial information from the dorsal stream, whereas the lateral entorhinal cortex (Brodmann area 28a) does so with the object-recognition information from the ventral stream. Furthermore, the

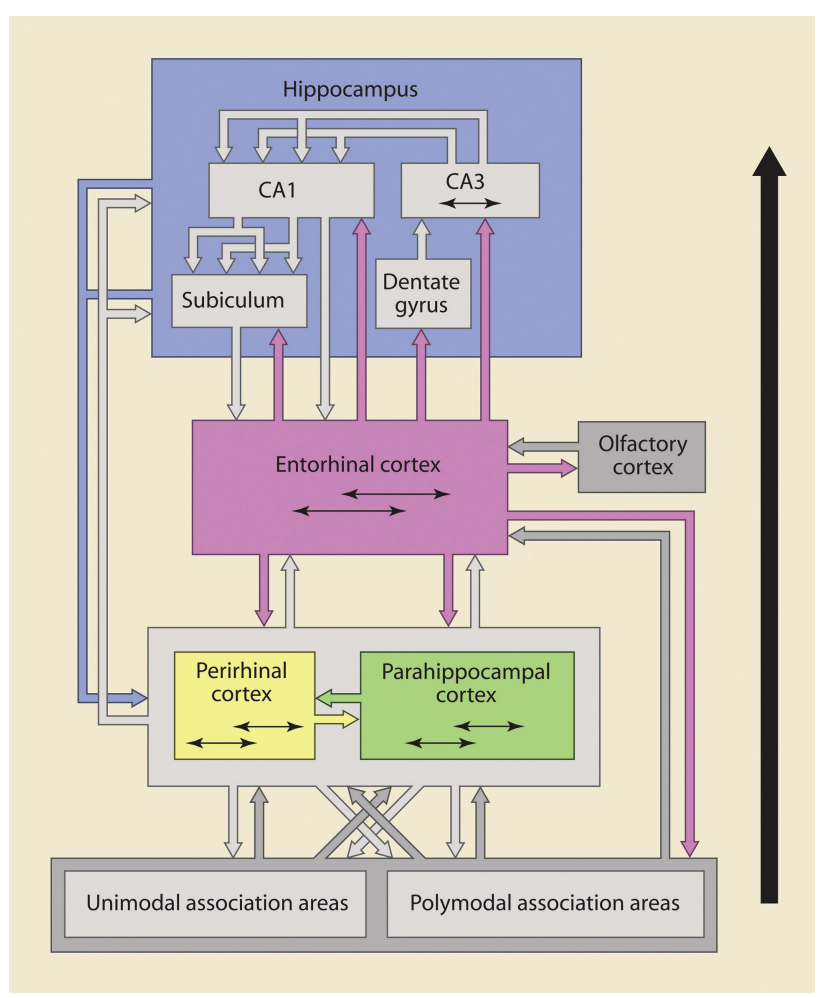

FIG 3. The net flow of information is from the perirhinal and parahippocampal cortices to the entorhinal cortex and then to the hippocampal formation, but considerable information processing occurs within and among the subregions of the parahippocampal gyrus before hippocampal formation involvement. Adapted with permission from Lavenex P, Amaral DG. Hippocampal-neocortical interaction: a hierarchy of associativity. Hippocampus 2000;10:420-30, John Wiley and Sons.

rhinal cortex functionally differentiates familiar and novel information input, where more familiar items are given fewer resources for encoding compared with new items. The rhinal cortex, therefore, functions as a gatekeeper of the declarative memory system by optimizing memory-encoding resources to novel information. ${ }^{12}$

In recent years, investigators have also postulated 2 cortical systems (anterior and posterior temporal) for memory-guided behavior involving the perirhinal and parahippocampal cortices. ${ }^{13}$ The anterior temporal system consists of the perirhinal cortex, temporopolar cortex, lateral orbital frontal cortex, and amygdala, while the posterior temporal system includes the parahippocampal cortex, retrosplenial cortex (Brodmann areas 29 and 30), anterior thalamic nuclei, mammillary bodies, pre- and parasubiculum, and components of the so-called default network, of which the retrosplenial cortex is a part, including the posterior cingulate gyrus, precuneus, angular gyrus, and ventral medial prefrontal cortex. The anterior system is more involved in object and face recognition, conceptual identity, and salience, while the posterior system focuses on scene recognition, location, trajectory, temporal context and order, and situations.

Semantic dementia involves more of the anterior temporal system, and patients often show the deficits in fine-grain object recognition. Alzheimer disease often involves more of the posterior temporal system and is frequently associated with deficits in 


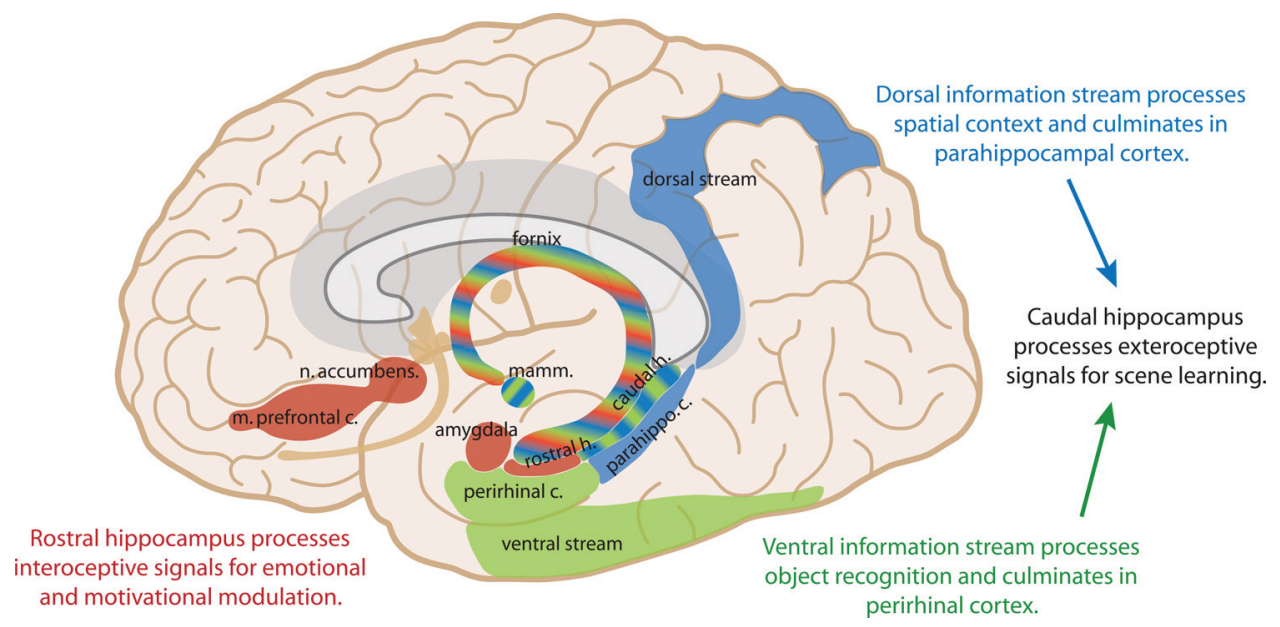

FIG 4. Medial temporal lobe processing of exteroceptive and interceptive signals for memory formation. The ventral stream from the occipital lobe projects information about object recognition, while the dorsal stream from the parietal lobe conveys spatial information. The rostral interceptive signals convey emotions and motivations.

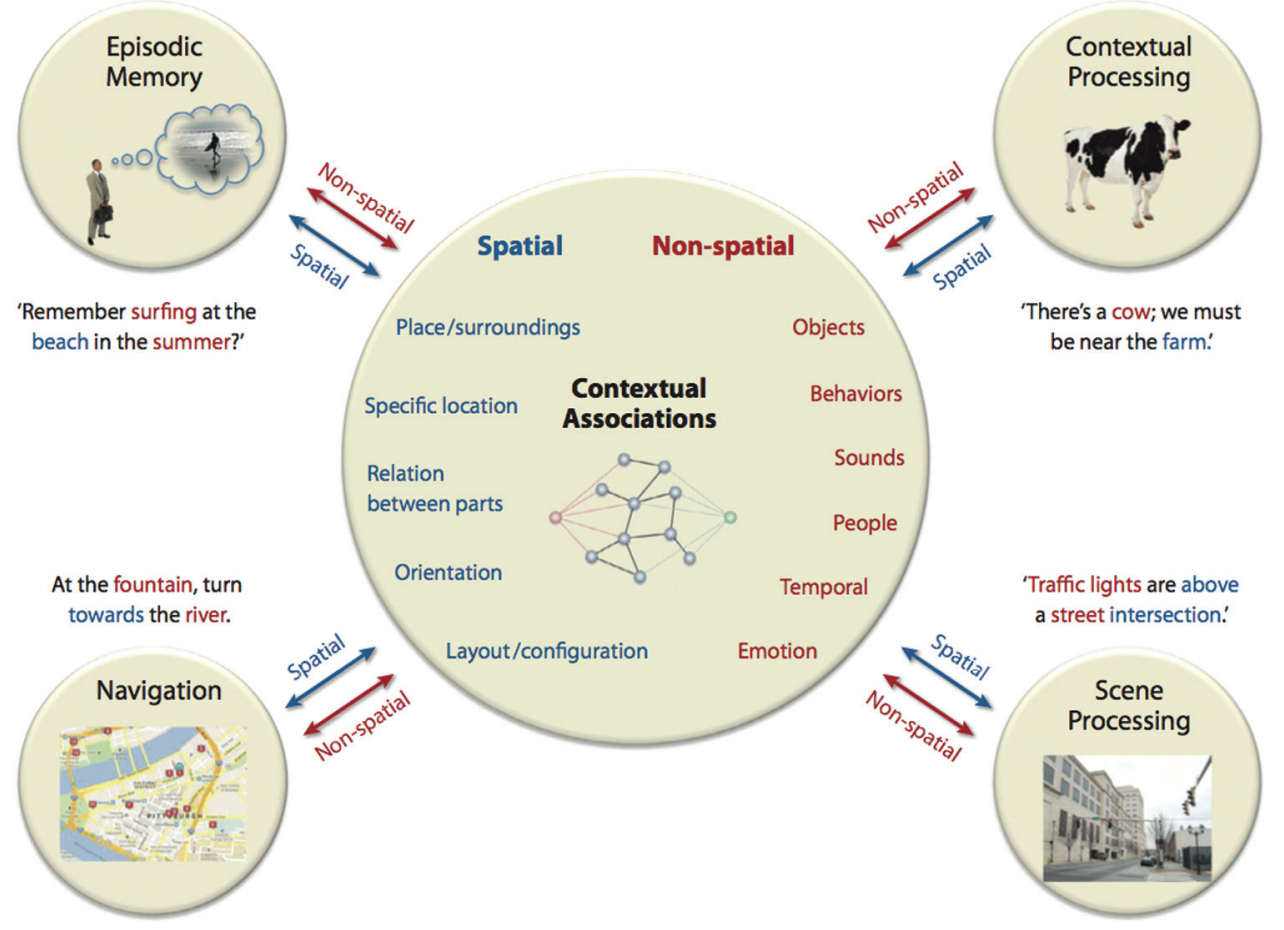

FIG 5. The parahippocampal cortex functions involve more than just spatial processing. The connections with the different areas of the frontal, parietal, and temporal lobes, including the default network, position the parahippocampal cortex as a critical component in processing contextual associations, which are fundamental aspects of higher cognitive functions. Adapted with permission from Aminoff EM, Kveraga K, Bar M. The role of the parahippocampal cortex in cognition. Trends Cogn Sci 2013;17:379-90, Elsevier.

scene discrimination. In this same fashion, detailed evaluation reveals that the parahippocampal cortex functions are much more complex than just the processing of spatial layout information. The parahippocampal cortex is part of a larger network that connects regions of the frontal, parietal, and temporal lobes. This includes auditory association areas of the superior temporal gyrus, the polymodal association areas (such as the retrosplenial cortex, lateral inferior parietal lobule, dorsal bank of the superior temporal sulcus), temporal pole, perirhinal cortex, parahippocampal cortex itself, entorhinal cortex, medial prefrontal cortex, dorsal lateral prefrontal cortex, orbital prefrontal cortex, in- sula, and so forth. It is best to think of the major role of the parahippocampal cortex as facilitating contextual associations, which are the principal elements underlying many higher level cognitive processes (Fig 5). ${ }^{14}$

In summary, the medial temporal lobe occupies a central position in the intersection of multiple neuronal networks. Its anatomic complexity within its subregions and with other cerebral structures reflects the multifaceted nature of memory. Its functions, however, are more than those associated with declarative memory and are now known to be wide-ranging and include higher level cognitive functions, especially with the connections 
with the retrosplenial cortex and default network as demonstrated by resting-state fMRI studies.

The next Functional Vignette will be the last installment on memory and will showcase clinical cases in which memory is affected when key anatomic structures are involved.

\section{ACKNOWLEDGMENTS}

The authors express our deepest gratitude to Jean Augustinack, $\mathrm{PhD}$, Harvard Medical School, Massachusetts General Hospital, Martinos Faculty, for her invaluable advice on the anatomy of the perirhinal and entorhinal cortices.

\section{REFERENCES}

1. Amaral DG. Introduction: what is where in the medial temporal lobe? Hippocampus 1999;9:1-6

2. Duvernoy HM, Cattin TP, Naidic C, et al. The Human Hippocampus: Functional Anatomy, Vascularization and Serial Sections with MRI. 3rd ed. Berlin: Springer-Verlag; 2005:232

3. Meunier M, Hadfield W, Bachevalier J, et al. Effects of rhinal cortex lesions combined with hippocampectomy on visual recognition memory in rhesus monkeys. J Neurophysiol 1996;75:1190-1205

4. Murray EA, Mishkin M. Visual recognition in monkeys following rhinal cortical ablations combined with either amygdalectomy or hippocampectomy. J Neurosci 1986;6:1991-2003
5. Liu Z, Richmond BJ, Murray EA, et al. DNA targeting of rhinal cortex D2 receptor protein reversibly blocks learning of cues that predict reward. Proc Natl Acad Sci U S A 2004;101:2336-41

6. Augustinack JC, Huber KE, Stevens AA, et al. Predicting the location of human perirhinal cortex, Brodmann's area 35, from MRI. Neuroimage 2013;64:32-42

7. Augustinack JC, van der Kouwe AJ, Fischl B. Medial temporal cortices in ex vivo magnetic resonance imaging. J Comp Neurol 2013; 521:4177-88

8. Feczko E, Augustinack JC, Fischl B, et al. An MRI-based method for measuring volume, thickness and surface area of entorhinal, perirhinal, and posterior parahippocampal cortex. Neurobiol Aging 2009;30;420-31

9. Lavenex P, Amaral DG. Hippocampal-neocortical interaction: a hierarchy of associativity. Hippocampus 2000;10:420-30

10. Purves D, Brannon E, Cabeza R, et al. Principles of Cognitive Neuroscience. Sunderland, MA: Sinauer Associates; 2008

11. Burgalossi A, Brecht M. Cellular, columnar and modular organization of spatial representations in medial entorhinal cortex. Curr Opin Neurobiol 2014;24:47-54

12. Fernández G, Tendolkar I. The rhinal cortex: 'gatekeeper' of the declarative memory system. Trends Cogn Sci 2006;10:358-62

13. Ranganath C, Ritchey M. Two cortical systems for memory-guided behavior. Nat Rev Neurosci 2012;13:713-26

14. Aminoff EM, Kveraga K, Bar M. The role of the parahippocampal cortex in cognition. Trends Cogn Sci 2013;17:379-90 